\title{
CXCR7 agonist TC14012 improves angiogenic function of endothelial progenitor cells via activating Akt/eNOS pathway and promotes ischemic angiogenesis in diabetic limb ischemia
}

\section{Kai Wang}

The First Affiliated Hospital of Wenzhou Medical University

Shiyue Sun

Wenzhou Medical University

Guigui Zhang

Wenzhou Medical University

Zixian Lu

Wenzhou Medical University

Hui Chen

Wenzhou Medical University

Xia Fan

Wenzhou Medical University

Chunjie Gu

Wenzhou Medical University

Xiaohong Pan

Wenzhou Medical University

Qian Lin

University of Louisville School of Medicine

Oscar Chen

University of Louisville School of Medicine

Lu Cai

University of Louisville School of Medicine

Xiaozhen Dai

Chengdu Medical College

Xiaoqing Yan ( $\triangle$ yanxiaoqing1128@163.com )

Wenzhou Medical University https://orcid.org/0000-0001-9113-5274

Yi Tan

University of Louisville School of Medicine 


\section{Research Article}

Keywords: TC14012, diabetes, endothelial progenitor cells, CXCR7, angiogenesis

Posted Date: January 25th, 2022

DOI: https://doi.org/10.21203/rs.3.rs-1261178/v1

License: (c) (i) This work is licensed under a Creative Commons Attribution 4.0 International License. Read Full License

Version of Record: A version of this preprint was published at Cardiovascular Drugs and Therapy on April 26th, 2022. See the published version at https://doi.org/10.1007/s10557-022-07337-9. 


\section{Abstract}

Purpose Endothelial progenitor cells (EPCs) play a critical role in repairing damaged vessels and triggering ischemic angiogenesis, but their number is reduced and function is impaired under diabetic conditions. Improving EPC function has been considered as a promising strategy to ameliorate diabetic vascular complications. In present study, we want to investigate whether and how CXCR7 agonist TC14012 promotes the angiogenic function of diabetic EPCs.

Methods High glucose (HG) treatment was used to mimic the hyperglycemia in diabetes. Tube formation, cell scratch recovery, and terminal deoxynucleotidyl transferase dUTP nick end labeling (TUNEL) assays were used to evaluate the angiogenic capability, cell migration and apoptosis of EPCs, respectively. Hind limb ischemia (HLI) model was used to appraise the ability of TC14012 in promoting diabetic ischemic angiogenesis in vivo.

Results HG treatment impaired EPC tube formation and migration, and induced EPC apoptosis and oxidative damage, while TC14012 rescued tube formation and migration, and prevented HG-induced apoptosis and oxidative damage of EPCs. Furthermore, these beneficial effects of TC14012 on EPCs were attenuated by specific siRNAs against CXCR7, validating that CXCR7 is a functional target of TC14012 in EPCs. Mechanistic studies demonstrated that HG treatment reduced CXCR7 expression in EPCs, impaired Akt and endothelial nitric oxide synthase (eNOS) phosphorylation and nitric oxide (NO) production; similarly, these signal impairments in HG-exposed EPCs could be rescued by TC14012. However, the protective effects of TC14012 on Akt and eNOS phosphorylation and NO production were almost completely abolished by siRNAs against CXCR7 or Akt specific inhibitor Wortmannin. More importantly, in vivo study showed that TC14012 administration enhanced blood perfusion recovery and angiogenesis in the ischemic hind limb and increased the EPC number in circulating of $d b / d b$ mice, demonstrating the capability of TC14012 in promoting EPC mobilization and ischemia angiogenic function.

Conclusion TC14012 can prevent EPCs from HG-induced dysfunction and apoptosis, improve eNOS activity and NO production via CXCR7/Akt signal pathway, and promote EPC mobilization and diabetic ischemia angiogenesis.

\section{Introduction}

Diabetes mellitus has become a rising epidemic worldwide. Type 2 diabetes, characterized by progressive insulin resistance and subsequent hyperglycemia $(1,2)$, accounts for more than $90 \%$ of the diabetic patients. Diabetic vascular complications, including diabetic limb ischemia, nephropathy, retinopathy, are the major cause of morbidity and mortality in diabetic patients and put huge burden on healthcare systems (3). Diabetic vascular complications were always initiated with endothelial dysfunction $(4,5)$, and accompanied with dysregulation of vascular remodeling and impaired/abnormal angiogenesis. 
Endothelial progenitor cells (EPCs) are the precursors of vascular endothelial cells (6). Accumulating evidence demonstrates that EPCs play a critical role in maintaining endothelial homeostasis and triggering angiogenesis (7-14). However, under diabetic conditions, the circulating EPC number was decreased and EPC function was impaired (15), which lead to attenuated repair of endothelium and ischemic angiogenesis, indicating that elevating circulating EPC number and/or improving EPC function is a potential strategy in the therapy of diabetic vascular complications.

Chemokine stromal cell derived factor 1 (SDF-1) is a key regulator of EPC mobilization, migration, retention and angiogenesis $(16,17)$. SDF- 1 exerts its biological function via its receptors $C-X-C$ chemokine receptor type 4 (CXCR4) and CXCR7 (15). SDF-1/CXCR4 axis plays a critical role in maintaining the quiescent state of EPCs in their bone marrow niche and specific antagonism of CXCR4 can mobilize EPCs from bone marrow into peripheral circulation to participate in vessel repair and angiogenesis (18). In addition, SDF-1/CXCR7 axis plays a primary role in maintaining EPC survival and function $(17,19)$. Moreover, our previous studies have demonstrated that CXCR7 expression in EPCs is downregulated under diabetic conditions, which is accompanied with EPC dysfunction and oxidative injury (15). More importantly, elevating CXCR7 expression improves survival and angiogenic function of EPCs and promotes blood perfusion in diabetic limb ischemia mouse model (15). These findings indicate the potential of targeting SDF-1/CXCR4/CXCR7 axis in ameliorating EPC dysfunction and promoting ischemic angiogenesis under diabetic conditions.

Cyclic peptide TC14012, a potent selective CXCR7 agonist (20-22), is also a strong antagonist against CXCR4 (20), has been reported to induce tube formation of human umbilical vein endothelial cells (23), reduce apoptosis in cardiovascular disease (24), and prevent lung epithelial damage and ameliorate fibrosis after bleomycin or hydrochloric acid induction (25). However, the effects of TC14012 on EPC angiogenic function and its therapeutic potential in diabetic ischemic injury remain elusive. Thus, the current study is aimed to investigate whether TC14012 can improve EPC function under diabetic conditions and clarify the underlying mechanism.

\section{Materials And Methods}

\section{EPC isolation, identification and treatment}

Human cord blood EPCs were isolated and cultured as described in our previous study (26). In brief, 50 $\mathrm{mL}$ human cord blood was obtained from umbilical vein of healthy post-partum women. Blood samples were diluted 1:1 with phosphate-buffered saline (PBS) and overlaid onto an equivalent volume of Histopaque 1077 (Sigma-Aldrich, St. Louis, MO). Mononuclear cells were isolated after density gradient centrifugation, then washed with Endothelial Cell Medium (ECM, ScienCell Research, Carlsbad, CA), and seeded into a culture dish $(\varphi=6 \mathrm{~cm})$ that pre-coated with fibronectin $\left(2 \mathrm{mg} / \mathrm{cm}^{2}\right)$ and cultured with ECM supplemented with $10 \%$ fetal bovine serum (FBS, Life Technologies, Austin, TX). After 3 days of culture, the unattached cells and debris were washed with PBS. The medium was changed daily during the first week and every 3 days thereafter. All protocols were approved by the Institutional Review Board of the 
First Affiliated Hospital of Wenzhou Medical University, and informed consents were all obtained from these subjects.

To mimic the hyperglycemia in diabetes in vitro, EPCs were treated with high glucose ( $\mathrm{HG}, 33 \mathrm{mmol} / \mathrm{L})$, and equivalent mannitol was used as osmotic control. After treatment for $24 \mathrm{~h}$, EPCs were harvested for further assays. For signal test, EPCs were harvested at 10 min after mannitol or HG with or without TC14012 (5 $\mu \mathrm{mol} / \mathrm{L}$, Cayman Chemical Company, Ann Arbor, MI) treatment.

\section{siRNA transfection}

To knockdown CXCR7 expression in EPCs, siRNAs against human CXCR7 or a Silencer Select Negative Control (GenePharma, Shanghai, China) were transfected into EPCs using GP-transfect-Mate (GenePharma, Shanghai, China). Three siRNAs against CXCR7 sequences are: CXCR7 siRNA-441 sense: GCUAUGACACGCACUGCUATT, antisense: UAGCAGUGCGUGUCAUAGCTT; CXCR7 siRNA-960 sense: GCAGCCGGAAGAUCAUCUUTT, antisense: AAGAUGAUCUUCCGGCUGCTT; and CXCR7 siRNA-1161 sense: GCUUCAUCAAUCGCAACUATT, antisense: UAGUUGCGAUUGAUGAAGCTT. The knockdown efficiency was detected by quantitative real-time polymerase chain reaction (qRT-PCR) and Western blot at $48 \mathrm{~h}$ post transfection, and the siRNA with the best knockdown efficiency was used in the following studies.

\section{Tube formation and cell scratch recovery assays}

Tube formation assay was adopted to investigate angiogenic capability of EPCs in vitro according to previous studies $(14,15)$ with minor modification. Briefly, a $\mu$-Slide angiogenesis plate (Ibidi, Shanghai, China) was coated with growth factor reduced Matrigel (10 $\mu \mathrm{L}$ per well, Corning). EPCs $\left(1.75 \times 10^{5}\right.$ cells/ml) were re-suspended in $50 \mu \mathrm{L}$ basal medium or medium containing $\mathrm{HG}$ in the presence or absence of TC14012, plated on the Matrigel, and incubated in cell incubator at $37^{\circ} \mathrm{C}$ with $5 \% \mathrm{CO}_{2}$ and saturation humidity. After $8 \mathrm{~h}$ culture, images of tube network in each well were taken using an inverted microscopy (Eclipse TI-S, Nikon, Japan) and total lengths of tube-like structures were quantified using ImageJ (NIH, Bethesda, Maryland).

Migration ability of EPCs was investigated by cell scratch recovery assay. Briefly, EPCs $\left(1.75 \times 10^{5} /\right.$ well $)$ were seeded onto 6-well plate and maintained to confluence. Thereafter, a scratch was made using pipette tips. EPCs were cultured in the basal medium with Man or HG containing with or without TC14012 for $24 \mathrm{~h}$. Mitomycin (10 ug/mL, Sigma-Aldrich, St. Louis, MO) was used to exclude the influence of cell proliferation. Scratches were recorded under a light microscopy (Leica DMI3000B, Wetzlar, Germany) equipped with a digital camera (Olympus DP25, Japan). The recovery of scratch was measured using ImageJ.

\section{Cell apoptosis}


One-step terminal deoxynucleotidyl transferase dUTP nick end labeling (TUNEL) apoptosis assay kit (Beyotime, Shanghai, China) was used to detect EPC apoptosis. According to the manufacturer's protocol, plated EPCs $\left(2 \times 10^{5}\right.$ cells/well) were rinsed with PBS and fixed with $4 \%$ paraformaldehyde. Then EPCs were incubated with immunostaining permeabilization buffer for $5 \mathrm{~min}$ and followed with reaction buffer at $37^{\circ} \mathrm{C}$ in dark for $1 \mathrm{~h}$. Fluorescent images were taken with inverted microscopy (Eclipse TI-S, Nikon, Japan). TUNEL positive nuclei were quantitatively counted manually, and cell apoptosis was presented as the ratio of TUNEL positive nuclei to the total nuclei recognized by 4',6-diamidino-2phenylindole (DAPI).

\section{Oxidative stress assay}

Reactive oxygen species (ROS) assay kit (Beyotime, Shanghai, China) was used to detect oxidative stress of EPCs. According to manufacturer's instruction, EPCs were incubated with dichlorofluorescin diacetate (DCF-DA) for $20 \mathrm{~min}$. Ten different fields per well were taken using a microscopy (Eclipse TI-S, Nikon, Japan) and the fluorescence intensity was analyzed using ImageJ.

\section{Western blot}

Western blot was performed as described in our previously studies $(14,15)$. Briefly, EPCs were lysed with cold radioimmunoprecipitation assay buffer (Cell Signaling Technology, Danvers, MA) at the indicated time points and clarified by centrifugation at $12,000 \mathrm{~g}$ at $4^{\circ} \mathrm{C}$ for $30 \mathrm{~min}$. Supernatants were collected and the protein concentration was determined by the Bradford assay (Thermo Fisher Scientific, Waltham, MA). Total $30 \mu \mathrm{g}$ proteins were prepared and mixed with loading buffer and dithiothreitol, and denatured by incubating at $95^{\circ} \mathrm{C}$ for $5 \mathrm{~min}$. The proteins were separated by a $10 \%$ SDS-PAGE and then transferred to nitrocellulose membranes (Merck Millipore, Danvers, MA). After blocking by $5 \%$ milk, membranes were incubated with primary antibodies (Cell Signaling Technology) against Akt $(1: 5,000)$ and phosphor-Akt (1:1,000), eNOS (1:5,000), phosphor-eNOS (1:1,000), 3-nitrotyrosine (3-NT, 1:1000) and 4-hydroxynonenal (4-HNE, 1:1000), at $4^{\circ} \mathrm{C}$ overnight. Then, the membranes were washed by Tris-buffered saline with Tween20 (TBST) for three times and incubated with appropriate horseradish peroxidase-conjugated secondary antibodies. Blots were visualized by ECL Plus (Solarbio, Beijing, China) and quantified using Quantity 5.2 (Bio-Rad, Hercules, CA).

\section{Nitric oxide (NO) assay}

The NO levels in EPC culture medium were tested by NO assay (Beyotime, Shanghai, China) following the manufacturer's instructions. 


\section{Diabetic hind limb ischemia (HLI) mice models and TC14012 treatment}

Male $d b / d b$ (BKS.Cg-Dock $\left.7^{m}+/+L e p r^{d b} / J\right)$ mice at the age of 8-12 weeks were obtained from The Jackson Laboratory (Bar Harbor, ME). HLI was performed as described previously $(8,14,15)$. Briefly, right hind limbs of the mice were shaved and operated under anesthetized condition with isoflurane $(1-3 \%$ isoflurane in $100 \%$ oxygen at a flowrate of $1 \mathrm{~L} / \mathrm{min}$ ). The entire right superficial femoral artery and vein were separated and ligated (6-0 silk sutures), cut, and excised with an electric coagulator, epidermis was sutured with 4-0 silk sutures. At $1 \mathrm{~h}$ after HLI surgery, TC14012 (10 mg/kg body weight) was injected intraperitoneally once and the same volume of PBS was used as vehicle control. Blood perfusion of the ischemic hind limb before surgery and at days $0,3,7,14,21,28$ after surgery was detected using a Pericam Perfusion Speckle Imager (PSI, Perimed Inc., Sweden), and presented as the ratio of the ischemic (right) to the contralateral non-ischemic (left) limb. All animal procedures were approved by the Institutional Animal Care and Use Committee of the University of Louisville, which conform to the Guide for the Care and Use of Laboratory Animals published by the US National Institutes of Health.

\section{Histological assessment}

Transverse sections of gastrocnemius and soleus muscles of ischemic hind limbs were cut and embedded in Optimal Cutting Temperature Compound for frozen sections. Antibody against CD31 (BD Pharmingen, San Jose, CA) was stained to recognize capillary of ischemic muscles, and 4',6-diamidino2phenylindole (Thermo Fisher Scientific, Waltham, MA) was used to recognize nuclei. Fluorescent fields were captured by fluorescence microscopy (DP74, Olympus). The capillary number was counted using Image $\mathrm{J}$ in randomly selected fields for a total of 20 different fields per section and 3 sections per animal. The capillary density was expressed as CD31 positive capillaries per field.

\section{Flow cytometry assay of circulating EPCs}

Whole blood of $d b / d b$ HLI mice treated with TC14012 (10 mg/kg body weight) was collected for flow cytometry analysis. According to our previous study $(14,15), 200 \mu \mathrm{L}$ peripheral blood was mixed with Lysing Solution (BD Biosciences, San Jose, CA) to lyse red blood cells. While blood cells were incubated with $5 \%$ bovine serum albumin (Sigma-Aldrich, St. Louis, MO) for $15 \mathrm{~min}$ for blockade of nonspecific binding and then stained with PE-conjugated anti-mouse CD34 and APC-conjugated anti-mouse VEGFR2 antibodies (BD Pharmingen, San Jose, CA) at room temperature for $1 \mathrm{~h}$. The same fluorescein-labeled isotype IgG was served as control to exclude the non-specific staining. After centrifugation and resuspension with $400 \mu \mathrm{L}$ PBS, cells were analyzed with a flow cytometer (NovoCyte, Agilent, USA), and data were analyzed using FlowJo. 


\section{qRT-PCR}

EPCs were lysed by Trizol (Tiangen Biotech, Beijing, China). Total RNA was extracted using a RNAsimple Total RNA Kit (Tiangen Biotech, Beijing, China) and reverse-transcribed using PrimeScript RT reagent Kit with gDNA Eraser (Takara Bio, Shiga, Japan) following the manufacturer's protocol. Primers of CXCR7 and $\beta$-actin were purchased from Genscript Biotechnology (Nanjing, China) and qRT-PCR was performed in duplication with a $20 \mu \mathrm{L}$ reaction system using a RT-PCR system (Takara, Shiga, Japan). The comparative cycle time (Ct) was used for determining fold differences, and $\beta$-actin was chosen as an endogenous reference $\left(2^{-\Delta \Delta C t}\right)$.

\section{Statistical analysis}

All data are presented as Mean \pm SD. Sample size for each study was detailed in figure legends.

Statistical analysis was performed using GraphPad Prism 5.0 with One-way or two-way ANOVA, followed by post-hoc Turkey's multiple comparisons. Statistical significance was considered as $\mathrm{P}<0.05$.

\section{Results}

\section{TC14012 protects EPCs from HG-induced angiogenic dysfunction and oxidative stress}

Matrigel tube formation assay was performed to test the protective effect of TC14012 on angiogenic function of EPCs in vitro. The results showed that HG treatment impaired tube formation of EPCs, while TC14012 obviously reversed this effect (Fig. 1A, B). Migration is an essential step in EPCs homing to the ischemic site and participating into vascular repair and angiogenesis. Similarly, scratch recovery assay showed that HG impaired EPC migration, while TC14212 preserved the migration ability of HG-treated EPCs (Fig. 1C, D). Furthermore, TC14012 also ameliorated HG-induced EPC apoptosis as determined by TUNEL staining (Fig. 1E, F).

As excessive generation of ROS is considered to be one of the major cause of diabetes-induced EPC dysfunction $(15,27)$, we investigated whether TC14012 prevents EPCs from HG-induced oxidative stress. DCF-DA staining showed that HG increased superoxide generation in EPCs, and this effect was completely abolished by TC14012 treatment (Fig. 2A, B). Moreover, the expression of oxidative stress biomarkers 3-NT (Fig. 2C) and 4-HNE (Fig. 2D) in EPCs were both elevated under HG condition, and reduced by TC14012 treatment, confirming TC14012 attenuation of HG-induced oxidative stress in EPCs. These findings demonstrate that TC14012 can prevent EPCs from HG-induced angiogenic dysfunction, apoptosis, and oxidative stress.

\section{CXCR7 knockdown abolishes the protective effects of TC14012 against HG-induced EPC dysfunction}

To test whether the protective effects of TC14012 against HG-induced EPC dysfunction relied on interaction with its receptor CXCR7, CXCR7 expression in EPCs was interfered by siRNA transfection (Supplementary Fig. S1A, B). Nonsense control-siRNA transfection did not affect TC14012 protecting HG- 
treated EPCs, while CXCR7-siRNA transfection nearly abolished the protective effects of TC14012 against HG-induced impairment of tube formation (Fig. 3A, B), migration (Fig. 3C, E), and apoptosis (Fig. 3D, F). Similarly, CXCR7-siRNA transfection also significantly impaired TC14012 protecting EPCs against HGinduced superoxide generation (Fig. 4A, B) and oxidative biomarkers 3-NT (Fig. 4C, D) and 4-HNE expression (Fig. 4C, E). Taken together, these findings indicate that CXCR7 plays a vital role in TC14012 protecting EPCs from HG-induced angiogenic dysfunction and oxidative stress.

\section{TC14012 prevents HG-induced impairment in Akt and eNOS activation and NO production in EPCs}

eNOS, an endothelial-cell-specific isoform of the NO producing enzyme, plays a vital role in maintaining the pro-angiogenic function of EPCs (28-30). In addition, Akt pathway was reported to mediate eNOS activation in endothelial cells (31). In the present study, we found that HG treatment inhibited CXCR7 expression (Fig. 5A, B), Akt (Fig. 5A, C) and eNOS (Fig. 5A, D) phosphorylation, and NO production (Fig. $5 E$ ) in EPCs, while these effects were significantly prevented by TC14012 treatment (Fig. 5A-E). These findings indicate that TC14012 preserves Akt and eNOS activation and NO production in HG-treated EPCs.

\section{CXCR7 mediates TC14012 preserving eNOS activation in HG-treated EPCs via activation of Akt pathway}

To confirm whether TC14012 activates Akt and eNOS via receptor CXCR7, CXCR7 expression was interfered using CXCR7 siRNA, and nonsense-siRNA was used as control (Fig. 6A, B). The results showed that control-siRNA did not affect the effect of TC14012 on Akt (Fig. 6A, C) and eNOS (Fig. 6A, D) phosphorylation and NO production (Fig. 6E). However, CXCR7-siRNA totally abolished the protective effects of TC14012 against HG-induced impairment in Akt and eNOS phosphorylation and NO production (Fig. 6A-E).

To further determine the critical role of Akt in TC14012/CXCR7 axis preserving eNOS activation, Akt signal was blocked by Wortmannin. The results showed that Wortmannin inhibited Akt phosphorylation (Fig. 7A, B) and abolished the protective effects of TC14012 against HG-induced impairment in eNOS phosphorylation (Fig. 7A, C). These results confirm that TC14012 preserving eNOS phosphorylation is mediated by Akt in HG-treated EPCs.

\section{TC14012 improves blood perfusion, ischemic angiogenesis, and mobilization of EPCs in diabetic HLI}

To determine the therapeutic potential of TC14012 in ameliorating diabetic EPC dysfunction in vivo, its capability in promoting ischemic angiogenesis and blood perfusion recovery was evaluated in HLI mouse model. The PSI scanning results showed that TC14012 administration significantly improved blood perfusion recovery since day 14 to day 28 post surgery compared with vehicle treat mice (Fig. 8A, B). Moreover, TC14012-treated mice had increased capillary density in both gastrocnemius and soleus muscles measured day 28 after HLI compared with vehicle treated mice (Fig. 8C, D), indicating that TC14012 can promote angiogenesis in ischemic tissue in diabetic mice. 
EPC mobilization is a critical step in ischemic angiogenesis (32). The flow cytometry results showed that TC14012-treated mice had higher circulating EPC number than that in vehicle group mice, indicating that TC14012 can also enhance EPC mobilization under diabetic ischemia conditions (Fig. 8E, F). Taken together, these in vivo results demonstrate that TC14012 can improve ischemic angiogenesis and blood perfusion recovery via promoting EPC mobilization and angiogenic function.

\section{Discussion}

In the present study, we demonstrate that TC14012 can improve tube formation, migration, and NO production capability of HG-treated EPCs in vitro and promote blood perfusion recovery in ischemic hind limb of diabetic mice via enhancing EPC mobilization and angiogenesis. Mechanistic study reveals that TC14012 alleviates oxidative stress and preserves angiogenic function of EPCs under diabetic conditions predominantly through activating CXCR7/Akt/eNOS pathways.

Emerging evidence indicates that CXCR7 is essential for regulating multiple cellular functional processes concerning endothelial hemostasis and angiogenesis $(15,26,33)$. Our previous studies also demonstrate that CXCR7 plays an important role in regulating EPC function (17), and elevating CXCR7 expression can reverse EPC dysfunction under diabetic conditions (15). However, this approach requires infusion of autologous or allogenic EPCs modified by CXCR7 transgene, which has practical limitations in clinical application. If therapeutic efficacy can be achieved using a CXCR7 specific agonist, it will increase the translational value of CXCR7 as a therapeutic target in the treatment of diabetic ischemia vascular diseases. In the present study, we found that TC14012, a specific agonist of CXCR7, protected EPCs against HG-induced impairment in tube formation, migration and cell survival (Fig. 1), and improved angiogenesis and blood perfusion recovery in diabetic HLI (Fig. 8). These findings are in line with previous studies showing that TC14012 alleviates myocardial infarction (34), promotes lung alveolar repair (25) and enhances angiogenesis after myocardial infarction (24) via activating CXCR7. These facts demonstrate a promising therapeutic potential of TC14012 for treatment of diabetic ischemia vascular diseases.

In addition, TC14012 accelerated angiogenesis and blood perfusion recovery is also associated with elevation of circulating EPC number in $d b / d b$ mice with HLI (Fig. 8E, F), indicating that TC14012 enhances EPC mobilization. Indeed, besides as an agonist of CXCR7, TC14012 can also function as an antagonist of CXCR4. SDF-1/CXCR4 axis plays a critical role in retaining the quiescent state of stem and progenitor cells in the bone marrow niche (35), and interfering SDF-1/CXCR4 interaction was considered as a feasible approach to mobilize EPCs. For example, AMD3100, a typical CXCR4 antagonist that used in clinic to mobilize stem cells for cell transplanting therapy, has been shown to accelerate blood flow restoration after $\mathrm{HLI}$ in diabetic mice via mobilizing EPCs $(36,37)$. Combining the benefits of TC14012 in improving EPC function as a CXCR7 agonist and promoting EPC mobilization as a CXCR4 antagonist to treat diabetic ischemia vascular diseases and other diabetic vascular complications in human patients needs further clinical validation. 
Elevated ROS was considered as one of the major causes of diabetic EPC dysfunction and impaired peripheral vascular regeneration $(15,38)$, and our previous studies found that overexpression of metallothionein (14) or activating nuclear factor (erythroid derived 2)-like 2 (15) diminished oxidative stress and protected EPCs against diabetes- or HG-induced injury. In line with these findings, our present findings also showed that TC14012 alleviated ROS in HG-treated EPCs (Fig. 2), and this beneficial effect on EPCs was abolished by CXCR7-siRNA (Fig. 4). These findings indicate that TC14012 ameliorates oxidative stress in HG-treated EPCs via its receptor CXCR7, which contributes to its preservation of EPC function under diabetic conditions.

NO regulates EPC mobilization from bone morrow niches into the peripheral circulation and participating in neovascularization. Decreased NO bioavailability has been proposed as one of the determinants of EPC dysfunction under diabetic conditions (39). The activity of eNOS, the major enzyme that regulates NO production in endothelial cells, was also repressed in diabetic EPCs. Conversely, NO replenishment is beneficial for diabetic ischemia tissue reperfusion (40). In addition, few reports have specifically linked CXCR7 and eNOS to EPC angiogenic function in diabetic ischemia. In the present study, our findings demonstrated that TC14012 treatment upregulated eNOS activity and NO production (Fig. 5A, D, E) and these effects were specifically mediated by CXCR7 in EPCs (Fig. 6A, D, E).

Although the reduction in NO bioavailability could be attributable to oxidative stress in diabetic EPCs, it also may be due to impairment of the protein kinase signaling cascades that are responsible for NO production, like Akt (39). We explored the role of Akt signal in TC14012 activating eNOS pathway in EPCs under diabetic conditions, and found that the phosphorylation of Akt and eNOS were all impaired by HG and rescued by TC14012 treatment (Fig. 5A, C, D) and blockade of Akt signal repressed the phosphorylation of eNOS (Fig. 7), indicating that TC14012 protecting diabetic EPCs is likely mediated by CXCR7/Akt/eNOS signal pathways.

In summary, TC14012 activation of CXCR7 improves EPC survival and function under diabetic conditions. This is because of improved anti-oxidative potency and NO production in EPCs, which is likely mediated by Akt activating eNOS phosphorylation. Pharmacological administration of TC14012 in vivo enhances diabetic ischemia angiogenesis, blood perfusion recovery and EPC mobilization, highlighting a clinical relevance for diabetic vascular ischemia treatment.

\section{Declarations}

\section{Acknowledgments}

This study was supported by a Junior Faculty Award (1-13-JF-53) from American Diabetes Association; National Key Research and Development Program of China (2017YFA0506000); the National Natural Science Foundation of China (81770305, 81873466), Natural Science Foundation of Zhejiang Province (LY22H070005, LY22H020005), Foundation for Distinguished Young Scholars of Sichuan Province (2019JDJQ0042), Key Research and Development Support Plan of Chengdu (2019-YF05-00275-SN), Fund of Development and Regeneration Key Laboratory of Sichuan Province (SYS18-04), Grant from 
Science and Technology Bureau of Wenzhou (Y20210010), and Basic Scientific Research Foundation of Wenzhou Medical University (KYYW201907).

\section{Funding}

This study was supported by a Junior Faculty Award (1-13-JF-53) from American Diabetes Association; National Key Research and Development Program of China (2017YFA0506000); the National Natural Science Foundation of China (81770305, 81873466), Natural Science Foundation of Zhejiang Province (LY22H070005, LY22H020005), Foundation for Distinguished Young Scholars of Sichuan Province (2019JDJQ0042), Key Research and Development Support Plan of Chengdu (2019-YF05-00275-SN), Fund of Development and Regeneration Key Laboratory of Sichuan Province (SYS18-04), Grant from Science and Technology Bureau of Wenzhou (Y20210010), and Basic Scientific Research Foundation of Wenzhou Medical University (KYYW201907).

\section{Competing Interests}

The authors have no relevant financial or non-financial interests to disclose

\section{Availability of data and material}

All data relevant to the study are included in the article or uploaded as supplementary information.

\section{Code availability}

Not applicable

\section{Author Contributions}

Kai Wang, Xiaoqing Yan and Yi Tan conceived and designed the study. Kai Wang, Shiyue Sun, Guigui Zhang, Zixian Lu, Hui Chen, Xia Fan Chunjie Gu, Xiaohong Pan, Oscar Chen and Qian Lin performed in vitro cell culture, cell biology, molecular biology experiments, and histology analysis. Kai Wang and Xiaozhen Dai performed in vivo therapeutic studies in mice. Kai Wang, Xiaoqing Yan and Yi Tan wrote the manuscript. Kai Wang and Lu Cai edited the manuscript with important intellectual content. Xiaoqing Yan and Yi Tan supervised this study.

\section{Ethics Approval}

All procedures performed in studies involving human participants were in accordance with the ethical standards of the Declaration of Helsinki. All protocols were approved by the Institutional Review Board of the First Affiliated Hospital of Wenzhou Medical University.

\section{Consent to Participate}

All samples were collected with written informed consent of the participants. 


\section{Consent for Publication}

Not applicable

\section{References}

1. Rodriguez-Gutierrez R, Gonzalez-Gonzalez J, Zuñiga-Hernandez J, McCoy R. Benefits and harms of intensive glycemic control in patients with type 2 diabetes. BMJ (Clinical research ed.). 2019;367:I5887. doi:10.1136/bmj.I5887.

2. Newman JD, Vani AK, Aleman JO, Weintraub HS, Berger JS, Schwartzbard AZ. The Changing Landscape of Diabetes Therapy for Cardiovascular Risk Reduction: JACC State-of-the-Art Review. J Am Coll Cardiol. 2018;72:1856-69. doi:10.1016/j.jacc.2018.07.071.

3. Chatterjee S, Khunti K, Davies MJ. Type 2 diabetes. Lancet. 2017;58:63. doi.

4. Shi Y, Vanhoutte PM. Macro- and microvascular endothelial dysfunction in diabetes. J Diabetes. 2017;9:434-49. doi:10.1111/1753-0407.12521.

5. Wasserman DH, Wang TJ, Brown NJ. The Vasculature in Prediabetes. Circ Res. 2018;122:1135-50. doi:10.1161/CIRCRESAHA.118.311912.

6. Chopra H, Hung MK, Kwong DL, Zhang CF, Pow EH N. Insights into Endothelial Progenitor Cells: Origin, Classification, Potentials, and Prospects. Stem Cells Int (2018) 2018: 9847015. doi: 10.1155/2018/9847015.

7. Aicher A, Heeschen C, Sasaki K-i, Urbich C, Zeiher AM, Dimmeler S. Low-energy shock wave for enhancing recruitment of endothelial progenitor cells: a new modality to increase efficacy of cell therapy in chronic hind limb ischemia. Circulation. 2006;114:2823-30. doi.

8. Yan X, Dai X, He L, Ling X, Shao M, Zhang C, et al. A Novel CXCR4 antagonist enhances angiogenesis via modifying the ischaemic tissue environment. J Cell Mol Med. 2017;21:2298-307. doi: $10.1111 / \mathrm{jcmm} .13150$.

9. Dai X, Yan X, Zeng J, Chen J, Wang Y, Chen J, et al. Elevating CXCR7 Improves Angiogenic Function of EPCs via Akt/GSK-3ß/Fyn-Mediated Nrf2 Activation in Diabetic Limb Ischemia. Circ Res. 2017;120:e7-23. doi:10.1161/circresaha.117.310619.

10. Williamson K, Stringer SE, Alexander MY. Endothelial progenitor cells enter the aging arena. Front Physiol. 2012;3:30. doi:10.3389/fphys.2012.00030.

11. Geng J, Wang L, Qu M, Song Y, Lin X, Chen Y, et al. Endothelial progenitor cells transplantation attenuated blood-brain barrier damage after ischemia in diabetic mice via HIF-1alpha. Stem Cell Res Ther. 2017;8:163. doi:10.1186/s13287-017-0605-3.

12. Grochot-Przeczek A, Kotlinowski J, Kozakowska M, Starowicz K, Jagodzinska J, Stachurska A, et al. Heme oxygenase- 1 is required for angiogenic function of bone marrow-derived progenitor cells: role in therapeutic revascularization. Antioxid Redox Signal. 2014;20:1677-92. doi:10.1089/ars.2013.5426. 
13. Xing Z, Zhao C, Liu H, Fan Y. Endothelial Progenitor Cell-Derived Extracellular Vesicles: A Novel Candidate for Regenerative Medicine and Disease Treatment. Adv Healthc Mater. 2020;9:e2000255. doi:10.1002/adhm.202000255.

14. Wang K, Dai X, He J, Yan X, Yang C, Fan X, et al. Endothelial Overexpression of Metallothionein Prevents Diabetes-Induced Impairment in Ischemia Angiogenesis Through Preservation of HIF1alpha/SDF-1/VEGF Signaling in Endothelial Progenitor Cells. Diabetes. 2020;69:1779-92. doi:10.2337/db19-0829.

15. Dai X, Yan X, Zeng J, Chen J, Wang Y, Chen J, et al. Elevating CXCR7 Improves Angiogenic Function of EPCs via Akt/GSK-3beta/Fyn-Mediated Nrf2 Activation in Diabetic Limb Ischemia. Circ Res. 2017;120:e7-23. doi:10.1161/CIRCRESAHA.117.310619.

16. Cun Y, Diao B, Zhang Z, Wang G, Yu J, Ma L, et al. Role of the stromal cell derived factor-1 in the biological functions of endothelial progenitor cells and its underlying mechanisms. Exp Ther Med. 2021;21:39. doi:10.3892/etm.2020.9471.

17. Dai $X$, Tan $Y$, Cai S, Xiong $X$, Wang $L, Y e Q$, et al. The role of CXCR7 on the adhesion, proliferation and angiogenesis of endothelial progenitor cells. J Cell Mol Med. 2011;15:1299-309. doi:10.1111/j.15824934.2011.01301.x.

18. Jujo K, Hamada H, Iwakura A, Thorne T, Sekiguchi H, Clarke T, et al. CXCR4 blockade augments bone marrow progenitor cell recruitment to the neovasculature and reduces mortality after myocardial infarction. Proc Natl Acad Sci U S A. 2010;107:11008-13. doi:10.1073/pnas.0914248107.

19. Yan X, Cai S, Xiong X, Sun W, Dai X, Chen S, et al. Chemokine receptor CXCR7 mediates human endothelial progenitor cells survival, angiogenesis, but not proliferation. J Cell Biochem. 2012;113:1437-46. doi:10.1002/jcb.24015.

20. Gravel S, Malouf C, Boulais PE, Berchiche YA, Oishi S, Fujii N, et al. The peptidomimetic CXCR4 antagonist TC14012 recruits beta-arrestin to CXCR7: roles of receptor domains. J Biol Chem. 2010;285:37939-43. doi:10.1074/jbc.C110.147470.

21. Ishizuka M, Harada M, Nomura S, Ko T, Ikeda Y, Guo J, et al. CXCR7 ameliorates myocardial infarction as a beta-arrestin-biased receptor. Sci Rep. 2021;11:3426. doi:10.1038/s41598-021-830225.

22. Ding BS, Cao Z, Lis R, Nolan DJ, Guo P, Simons M, et al. Divergent angiocrine signals from vascular niche balance liver regeneration and fibrosis. Nature. 2014;505:97-102. doi:10.1038/nature12681.

23. Zhang M, Qiu L, Zhang $Y, X u$, Zheng JC, Jiang L. CXCL12 enhances angiogenesis through CXCR7 activation in human umbilical vein endothelial cells. Sci Rep. 2017;7:8289. doi:10.1038/s41598-01708840-y.

24. Zhang S, Yue J, Ge Z, Xie Y, Zhang M, Jiang L. Activation of CXCR7 alleviates cardiac insufficiency after myocardial infarction by promoting angiogenesis and reducing apoptosis. Biomed Pharmacother. 2020;127:110168. doi:10.1016/j.biopha.2020.110168.

25. Cao Z, Lis R, Ginsberg M, Chavez D, Shido K, Rabbany SY, et al. Targeting of the pulmonary capillary vascular niche promotes lung alveolar repair and ameliorates fibrosis. Nat Med. 2016;22:154-62. 
doi:10.1038/nm.4035.

26. Fan X, He L, Dai Q, He J, Chen X, Dai X, et al. Interleukin-1 beta augments the angiogenesis of endothelial progenitor cells in an NF-kappaB/CXCR7-dependent manner. J Cell Mol Med. 2020;24:5605-14. doi:10.1111/jcmm.15220.

27. Zhang Z, Wang S, Zhou S, Yan X, Wang Y, Chen J, et al. Sulforaphane prevents the development of cardiomyopathy in type 2 diabetic mice probably by reversing oxidative stress-induced inhibition of LKB1/AMPK pathway. J Mol Cell Cardiol. 2014;77:42-52. doi:10.1016/j.yjmcc.2014.09.022.

28. Huang CC, Kuo HM, Wu PC, Cheng SH, Chang TT, Chang YC, et al. Soluble delta-like 1 homolog (DLK1) stimulates angiogenesis through Notch1/Akt/eNOS signaling in endothelial cells. Angiogenesis. 2018;21:299-312. doi:10.1007/s10456-018-9596-7.

29. Icli B, Wu W, Ozdemir D, Li H, Cheng HS, Haemmig S, et al. MicroRNA-615-5p Regulates Angiogenesis and Tissue Repair by Targeting AKT/eNOS (Protein Kinase B/Endothelial Nitric Oxide Synthase) Signaling in Endothelial Cells. Arterioscler Thromb Vasc Biol. 2019;39:1458-74. doi:10.1161/atvbaha.119.312726.

30. Boopathy GTK, Kulkarni M, Ho SY, Boey A, Chua EWM, Barathi VA, et al. Cavin-2 regulates the activity and stability of endothelial nitric-oxide synthase (eNOS) in angiogenesis. J Biol Chem. 2017;292:17760-76. doi:10.1074/jbc.M117.794743.

31. Dimmeler S, Fleming I, Fisslthaler B, Hermann C, Busse R, Zeiher AM. Activation of nitric oxide synthase in endothelial cells by Akt-dependent phosphorylation. Nature. 1999;399:601-5. doi:10.1038/21224.

32. Sainz J, Sata M. CXCR4, a key modulator of vascular progenitor cells. Arterioscler Thromb Vasc Biol. 2007;27:263-5. doi:10.1161/01.ATV.0000256727.34148.e2.

33. Hao H, Hu S, Chen H, Bu D, Zhu L, Xu C, et al. Loss of Endothelial CXCR7 Impairs Vascular Homeostasis and Cardiac Remodeling After Myocardial Infarction: Implications for Cardiovascular Drug Discovery. Circulation. 2017;135:1253-64. doi:10.1161/CIRCULATIONAHA.116.023027.

34. Ishizuka M, Harada M, Nomura S, Ko T, Ikeda Y, Guo J, et al. CXCR7 ameliorates myocardial infarction as a $\beta$-arrestin-biased receptor. Sci Rep. 2021;11:3426. doi:10.1038/s41598-021-83022-5.

35. Lapidot T, Dar A, Kollet O How do stem cells find their way home? Blood (2005) 106: 1901-10. doi: 10.1182/blood-2005-04-1417.

36. Tan Y, Li Y, Xiao J, Shao H, Ding C, Arteel GE, et al. A novel CXCR4 antagonist derived from human SDF-1beta enhances angiogenesis in ischaemic mice. Cardiovasc Res. 2009;82:513-21. doi:10.1093/cvr/cvp044.

37. Jiao C, Fricker S, Schatteman GC. The chemokine (C-X-C motif) receptor 4 inhibitor AMD3100 accelerates blood flow restoration in diabetic mice. Diabetologia. 2006;49:2786-9. doi:10.1007/s00125-006-0406-1.

38. Sorrentino SA, Bahlmann FH, Besler C, Muller M, Schulz S, Kirchhoff N, et al. Oxidant stress impairs in vivo reendothelialization capacity of endothelial progenitor cells from patients with type 2 diabetes 
mellitus: restoration by the peroxisome proliferator-activated receptor-gamma agonist rosiglitazone. Circulation. 2007;116:163-73. doi:10.1161/CIRCULATIONAHA.106.684381.

39. Hamed S, Brenner B, Roguin A. Nitric oxide: a key factor behind the dysfunctionality of endothelial progenitor cells in diabetes mellitus type-2. Cardiovasc Res. 2011;91:9-15. doi:10.1093/cvr/cvq412.

40. Ambasta RK, Kohli H, Kumar P. Multiple therapeutic effect of endothelial progenitor cell regulated by drugs in diabetes and diabetes related disorder. J Transl Med. 2017;15:185. doi:10.1186/s12967017-1280-y.

\section{Figures}


A

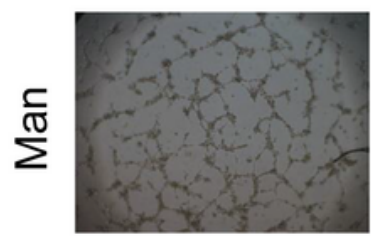

운

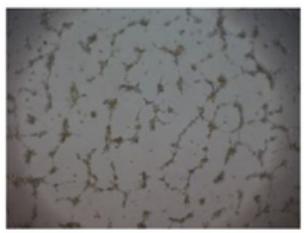

C

Oh

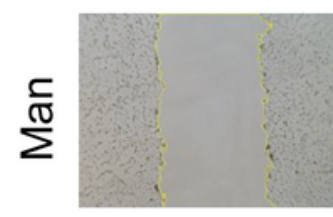

0
+
+
$\stackrel{0}{0}$
$\Sigma$

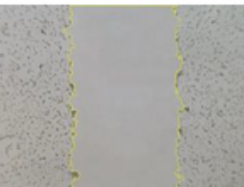

오
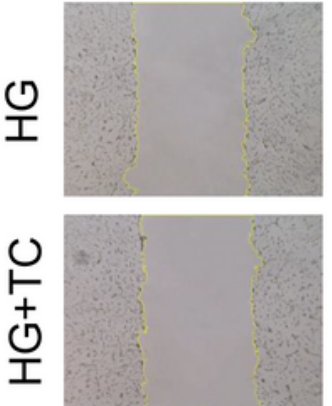

$\mathrm{F}$

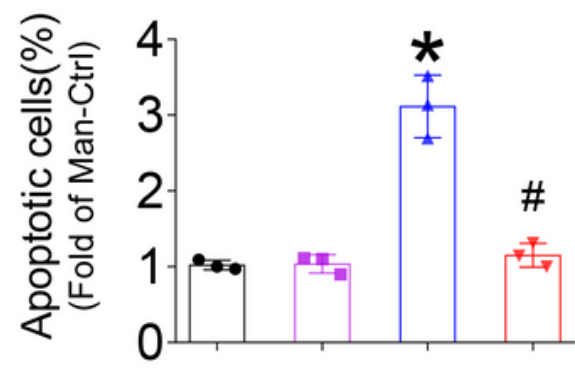

B

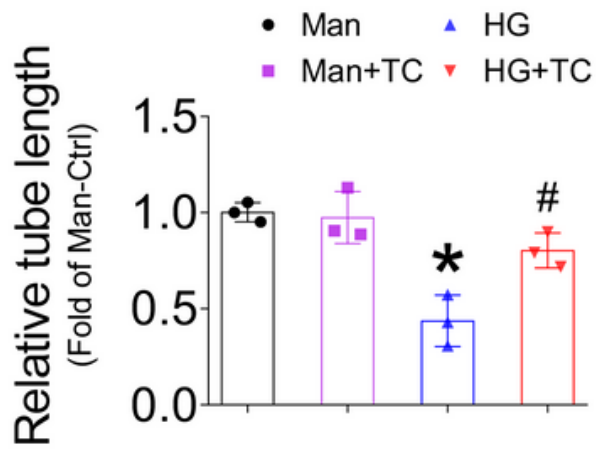

D

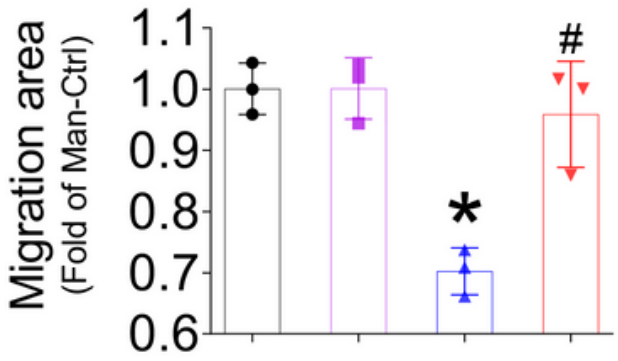

E

TUNEL-Cy3 DAPI

Merge
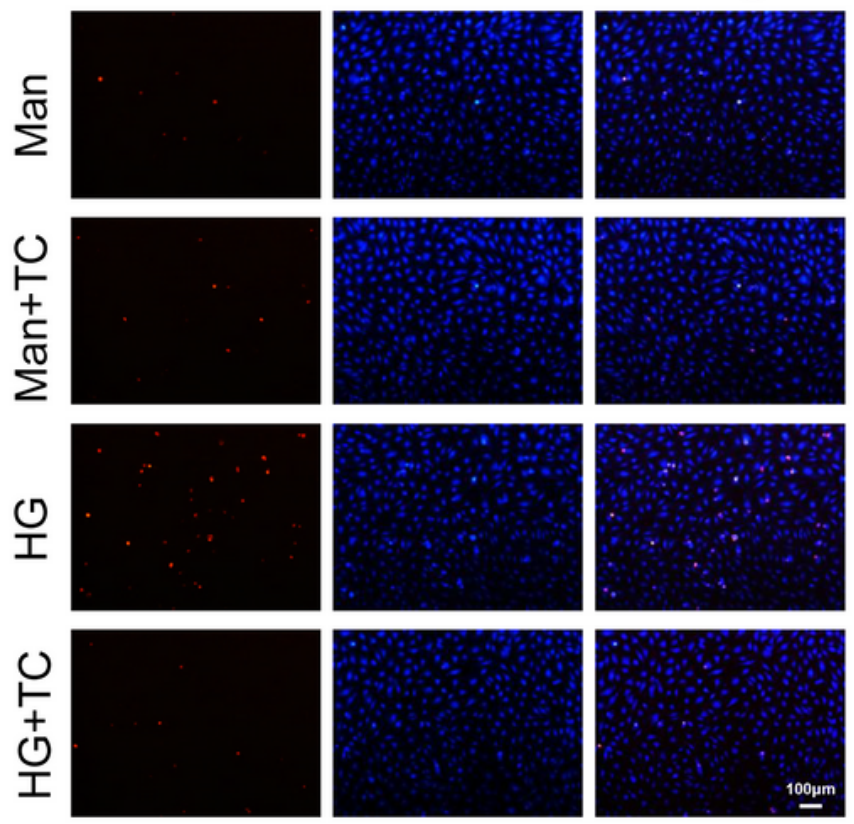

Figure 1

TC14012 protects EPCs from HG-induced angiogenic dysfunction and apoptosis. EPCs were exposed to high glucose (HG, $33 \mathrm{mmol} / \mathrm{L}$ ) with or without TC14012 (TC, $5 \mu \mathrm{mol} / \mathrm{L}$ ) treatment for $24 \mathrm{~h}$, the equivalent concentration of mannitol (Man) was used as osmotic control (Ctrl). The angiogenic function of EPCs was determined by tube formation assay (A) and the tube length was quantified using Image $(B)$. The migration capability of EPCs was evaluated by cell scratch recovery assay (C) and the cell scratch 
recovery area was quantified using ImageJ (D). The apoptosis of EPCs was detected by TUNEL staining $(\mathrm{E})$ and ratio of TUNEL positive nuclei to total nuclei recognized by DAPI was calculated (F). Data were normalized to Man group and expressed as Mean \pm SD. ${ }^{*} P<0.05$ vs. Man; ${ }^{\#} P<0.05$ vs. HG.
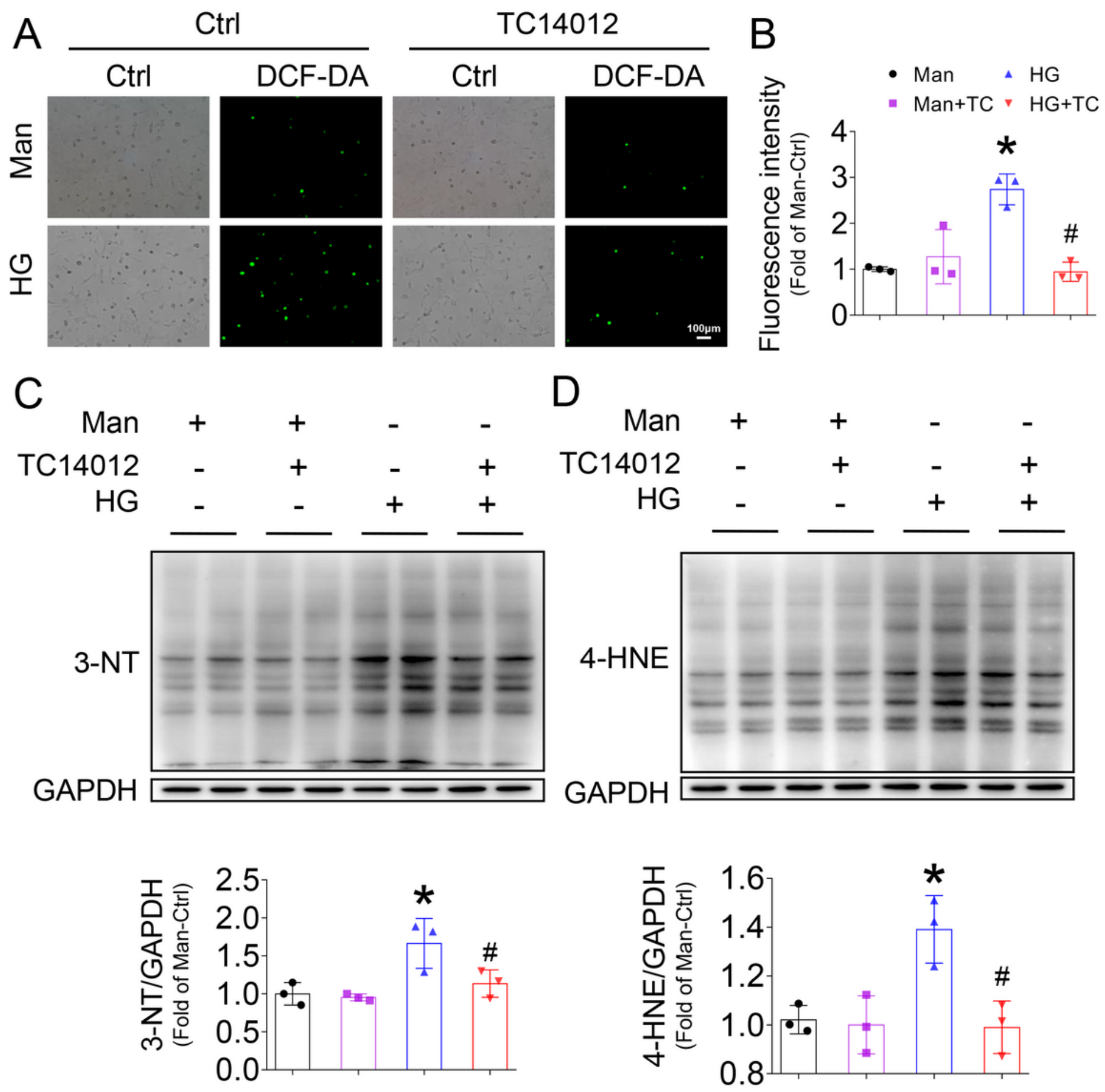

Figure 2

TC14012 attenuates HG-induced oxidative damage in EPCs. EPCs were exposed to high glucose (HG, 33 $\mathrm{mmol} / \mathrm{L}$ ) with or without TC14012 (TC, $5 \mu \mathrm{mol} / \mathrm{L})$ treatment for $24 \mathrm{~h}$, the equivalent concentration of mannitol (Man) was used as osmotic control (Ctrl). The reactive oxygen species (ROS) generation was 
detected by dichlorofluorescin diacetate (DCF-DA) staining (A) and the fluorescent intensity of ROS was quantified using ImageJ (B). The expression levels of the oxidative damage markers 3-nitroryrosine (3-NT, C) and 4-hydroxynonenal (4-HNE, D) in EPCs were detected by Western blot and GAPDH was used as loading control. Three independent experiments were performed. Data were normalized to Man group and expressed as Mean $\pm \mathrm{SD}$. ${ }^{\star} P<0.05$ vs. Man; $\# P<0.05$ vs. HG.

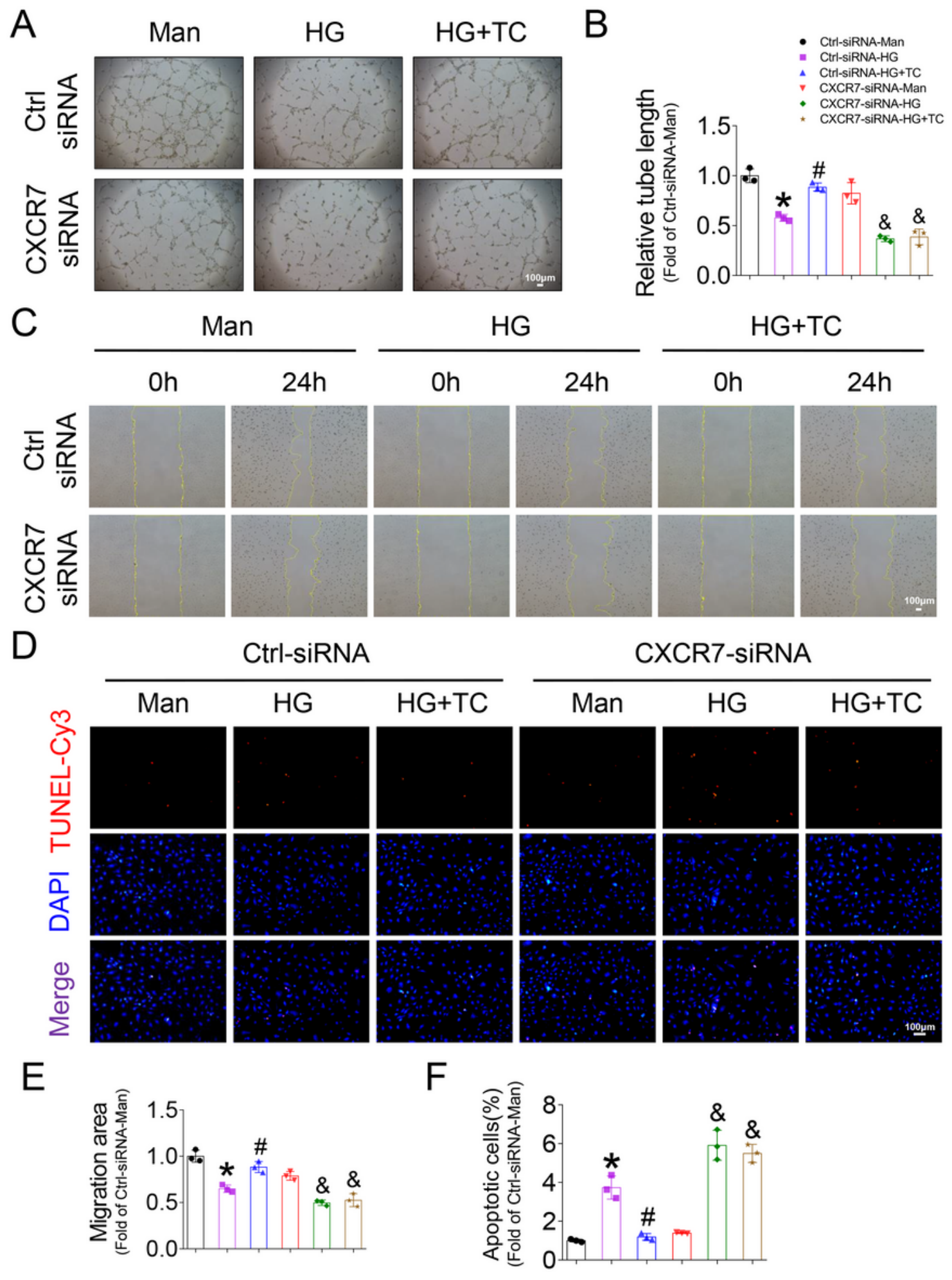

Figure 3 


\section{Knockdown of CXCR7 abolishes TC14012 protecting EPCs from HG-induced angiogenic dysfunction and}

apoptosis. EPCs were transfected with the specific siRNA against CXCR7 for $48 \mathrm{~h}$, the Silencer Select Negative Control was used as control siRNA (Ctrl-siRNA). Then the transfected EPCs were exposed to high glucose (HG, $33 \mathrm{mmol} / \mathrm{L})$ for additional $24 \mathrm{~h}$ with or without TC14012 (TC, $5 \mu \mathrm{mol} / \mathrm{L})$ treatment, the equivalent concentration of mannitol (Man) was used as osmotic control (Ctrl). The angiogenic function of EPCs was evaluated by tube formation assay (A) and the tube length was quantified using ImageJ (B). The migration capability was evaluated by cell scratch recovery assay (C) and the scratch recovery was quantified using ImageJ (E). The apoptosis of EPCs was detected by a TUNEL staining (D) and the ratio of TUNEL positive nuclei to the total nuclei recognized by DAPI was calculated (F). Three independent experiments were performed. Data were normalized to Ctrl-siRNA-Man group and expressed as Mean \pm SD. ${ }^{*} P<0.05$ vs. Ctrl-siRNA-Man; $\# P<0.05$ vs. Ctrl-siRNA-HG; $\& P<0.05$ vs. Ctrl-siRNA-HG+TC. 
A

Man

HG

$\mathrm{HG}+\mathrm{TC}$
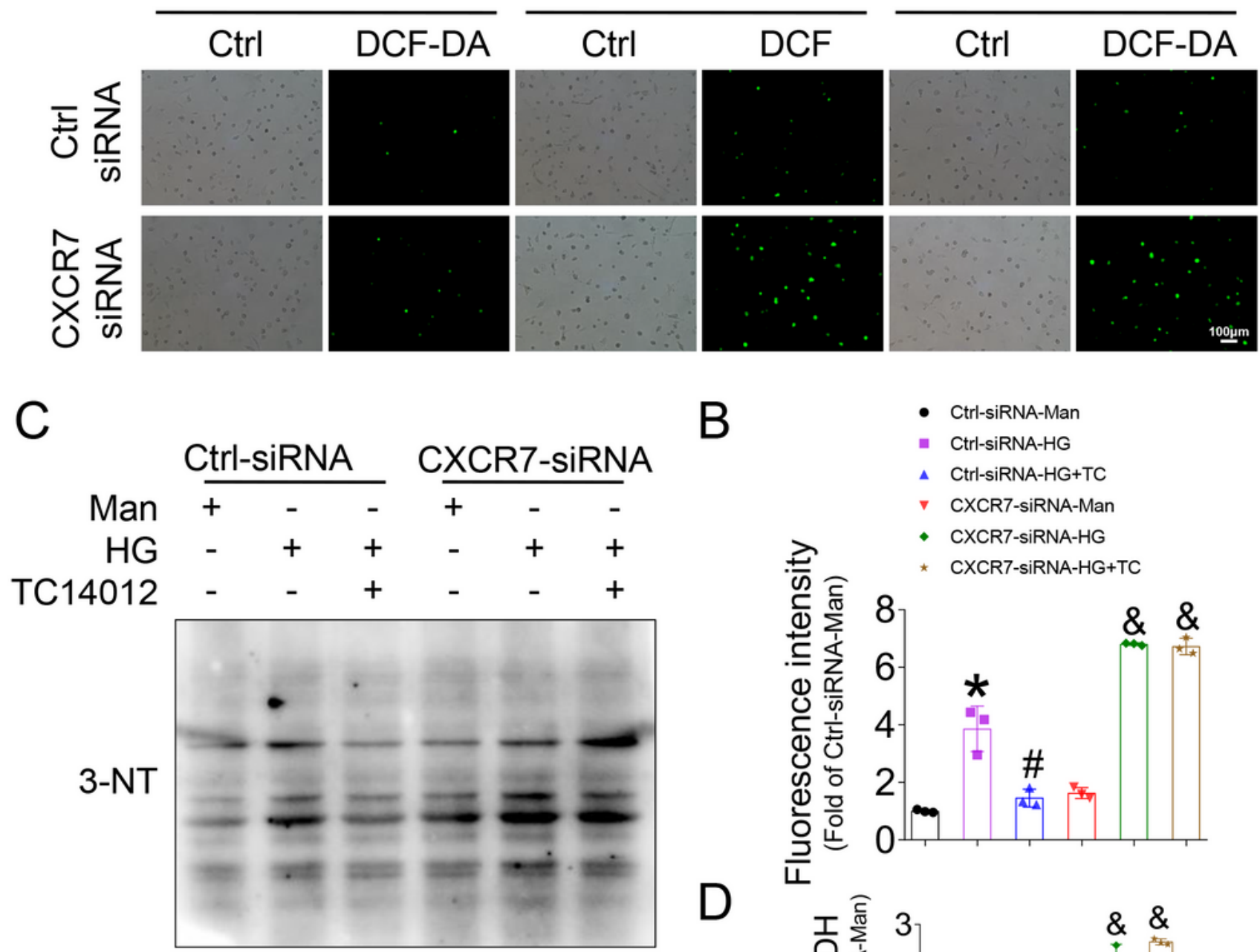

B
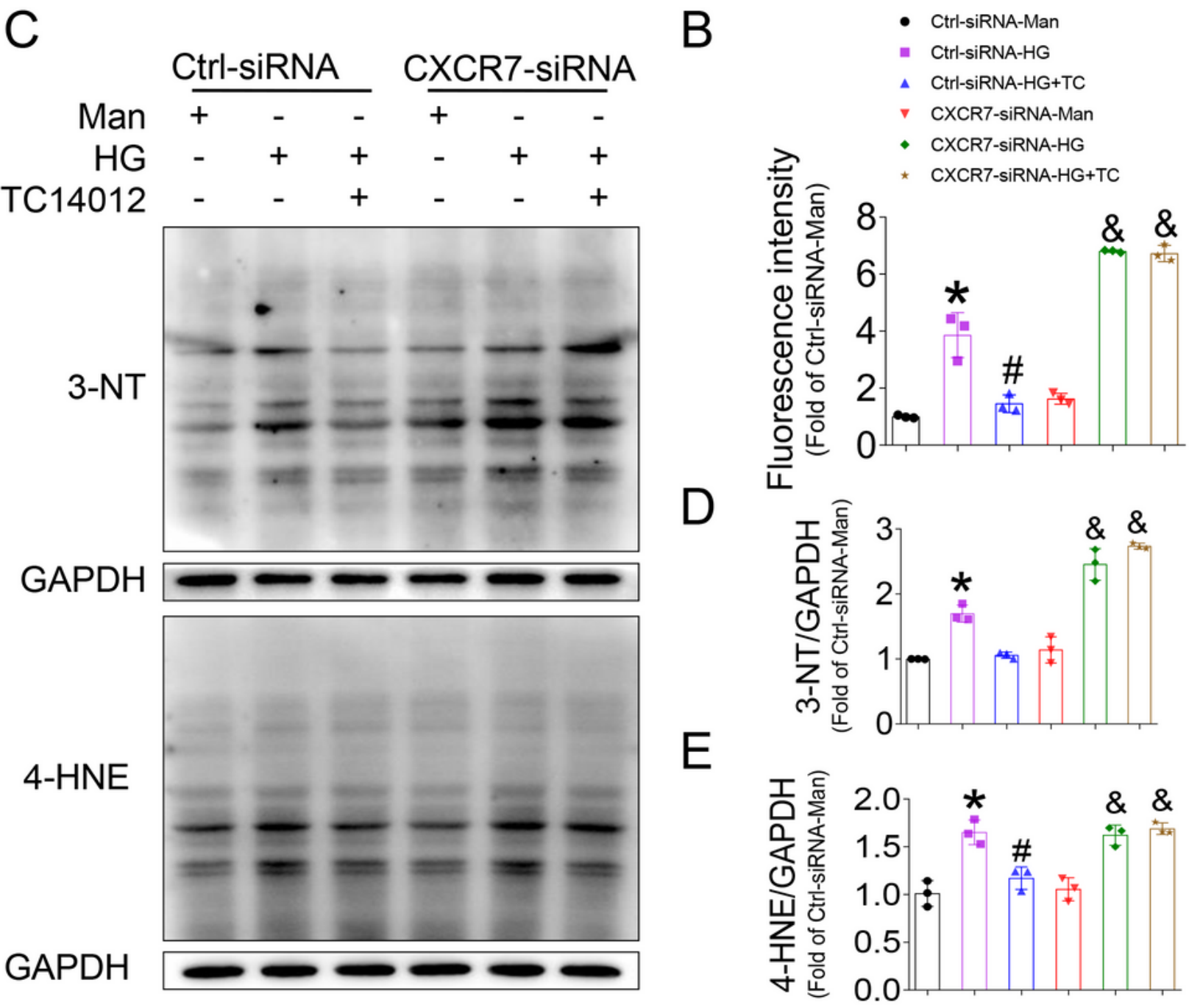

E

Figure 4

Knockdown of CXCR7 abolishes TC14012 protecting EPCs from HG-induced oxidative damage. EPCs were transfected with the specific siRNA against CXCR7 for $48 \mathrm{~h}$, the Silencer Select Negative Control was used as control siRNA (Ctrl-siRNA). Then the transfected EPCs were exposed to high glucose (HG, 33 $\mathrm{mmol} / \mathrm{L}$ ) for additional $24 \mathrm{~h}$ with or without TC14012 (TC, $5 \mu \mathrm{mol} / \mathrm{L}$ ) treatment, the equivalent concentration of mannitol (Man) was used as osmotic control (Ctrl). The reactive oxygen species (ROS) 
generation was detected by dichlorofluorescin diacetate (DCF-DA) staining (A) and the fluorescent intensity of ROS was quantified using Image (B). The expression levels of the oxidative damage markers 3-nitroryrosine (3-NT, C, D) and 4-hydroxynonenal (4-HNE, C, E) in EPCs were detected by Western blot and GAPDH was used as loading control. Three independent experiments were performed. Data were normalized to Ctrl-siRNA-Man group and expressed as Mean \pm SD. ${ }^{*} P<0.05$ vs. Ctrl-siRNA-Man; $\# P<0.05$ vs. Ctrl-siRNA-HG; \&P<0.05 vs. Ctrl-siRNA-HG+TC.

A

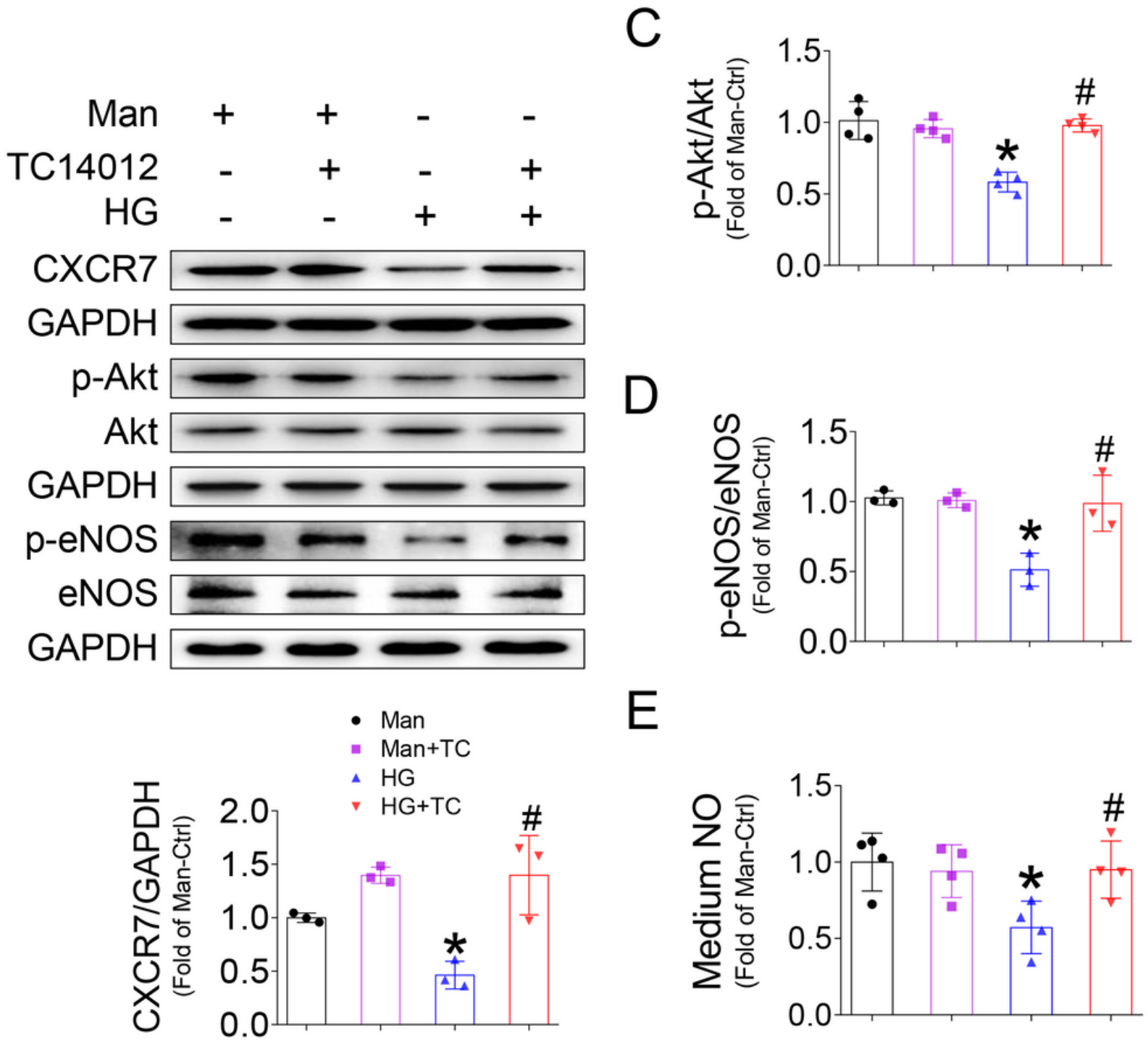

Figure 5

TC14012 prevents HG-induced Akt and eNOS inactivation and NO reduction in EPCs. EPCs were exposed to high glucose ( $\mathrm{HG}, 33 \mathrm{mmol} / \mathrm{L})$ with or without TC14012 (TC, $5 \mu \mathrm{mol} / \mathrm{L})$ treatment for $10 \mathrm{~min}$, the equivalent concentration of mannitol (Man) was used as osmotic control (Ctrl). The expression of CXCR7, and phosphorylation of Akt and eNOS were evaluated by Western blot (A-D) and GAPDH was used as loading control. The level of NO was evaluated by a NO assay kit (E). Three independent experiments 
were performed. Data were normalized to Ctrl-siRNA-Man group and expressed as Mean \pm SD. ${ }^{\star} P<0.05$ vs. Man, $\# P<0.05$ vs. HG.

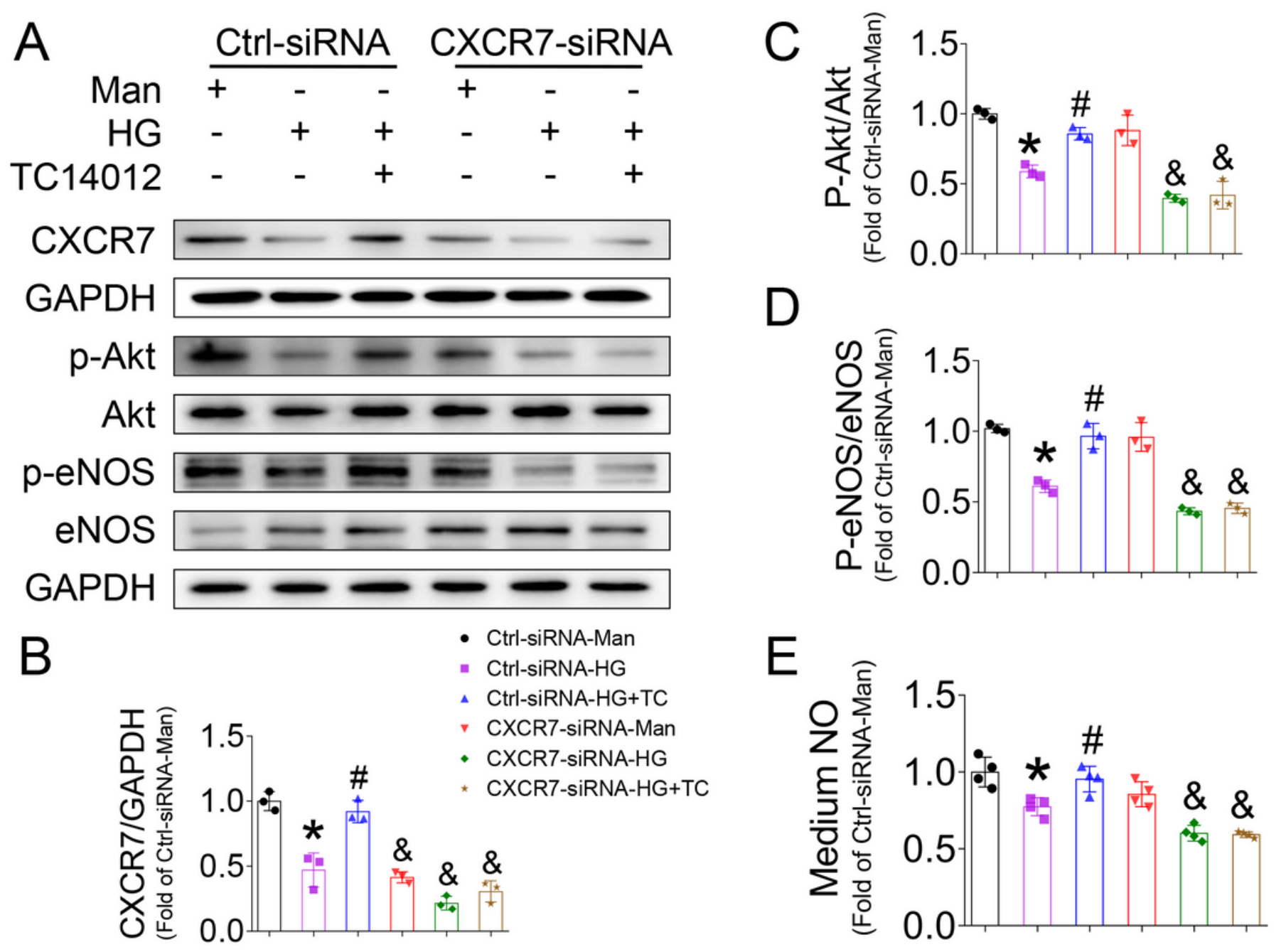

Figure 6

Knockdown of CXCR7 abolishes TC14012 preventing HG-induced Akt and eNOS inactivation and NO reduction in EPCs. EPCs were transfected with the specific siRNA against CXCR7 for $48 \mathrm{~h}$, the Silencer Select Negative Control was used as control siRNA (Ctrl-siRNA). Then the transfected EPCs were exposed to high glucose (HG, $33 \mathrm{mmol} / \mathrm{L})$ for additional $10 \mathrm{~min}$ with or without TC14012 (TC, $5 \mu \mathrm{mol} / \mathrm{L})$ treatment, the equivalent concentration of mannitol (Man) was used as osmotic control (Ctrl). The expression of CXCR7 (A, B), and phosphorylation of Akt (A, C) and eNOS (A, D) were evaluated by Western blot and GAPDH was used as loading control. The level of NO was evaluated by NO assay (E). Three independent experiments were performed. Data were normalized to Man group and expressed as Mean \pm SD. ${ }^{*} P<0.05$ vs. Ctrl-siRNA-Man; $\# P<0.05$ vs. Ctrl-siRNA-HG; $\& P<0.05$ vs. Ctrl-siRNA-HG+TC. 

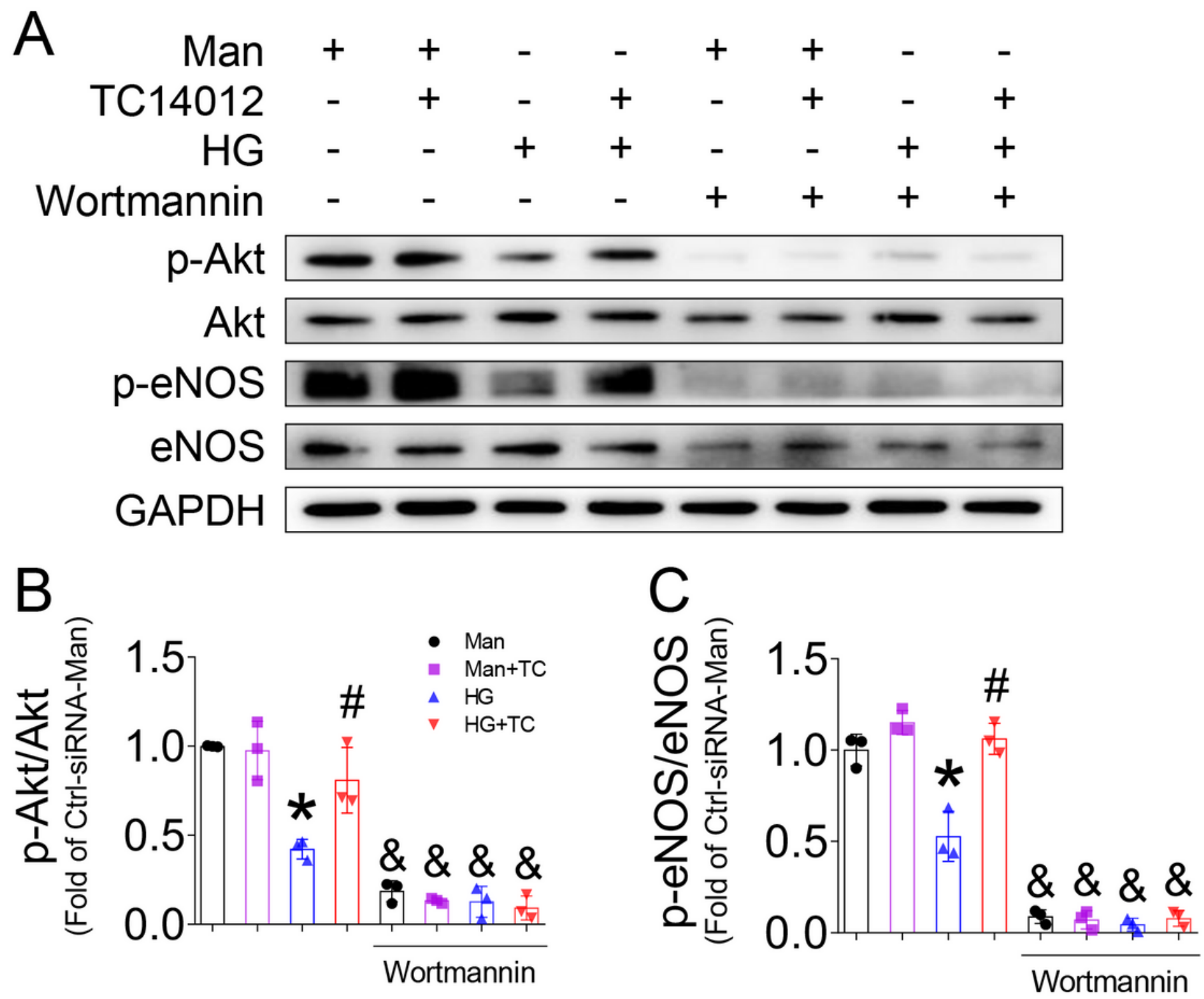

Figure 7

Akt inhibitor Wortmannin abolishes TC14012 preventing HG-induced eNOS inactivation in EPCs. EPCs were exposed to high glucose (HG, $33 \mathrm{mmol} / \mathrm{L})$ with or without TC14012 (TC, $5 \mu \mathrm{mol} / \mathrm{L})$ for $10 \mathrm{~min}$, the equivalent concentration of mannitol (Man) was used as osmotic control (Ctrl). The phosphorylation of Akt $(A, B)$ and eNOS (A, C) in EPCs with or without Wortmannin $(200 \mathrm{nmol} / \mathrm{L})$ pretreatment was evaluated by Western blot, and GAPDH was used as loading control. Three independent experiments were performed. Data were normalized to Ctrl-siRNA-Man group and expressed as Mean \pm SD. ${ }^{\star} P<0.05$ vs. Man; \#P<0.05 vs. HG; \&P<0.05 vs. HG+TC. 


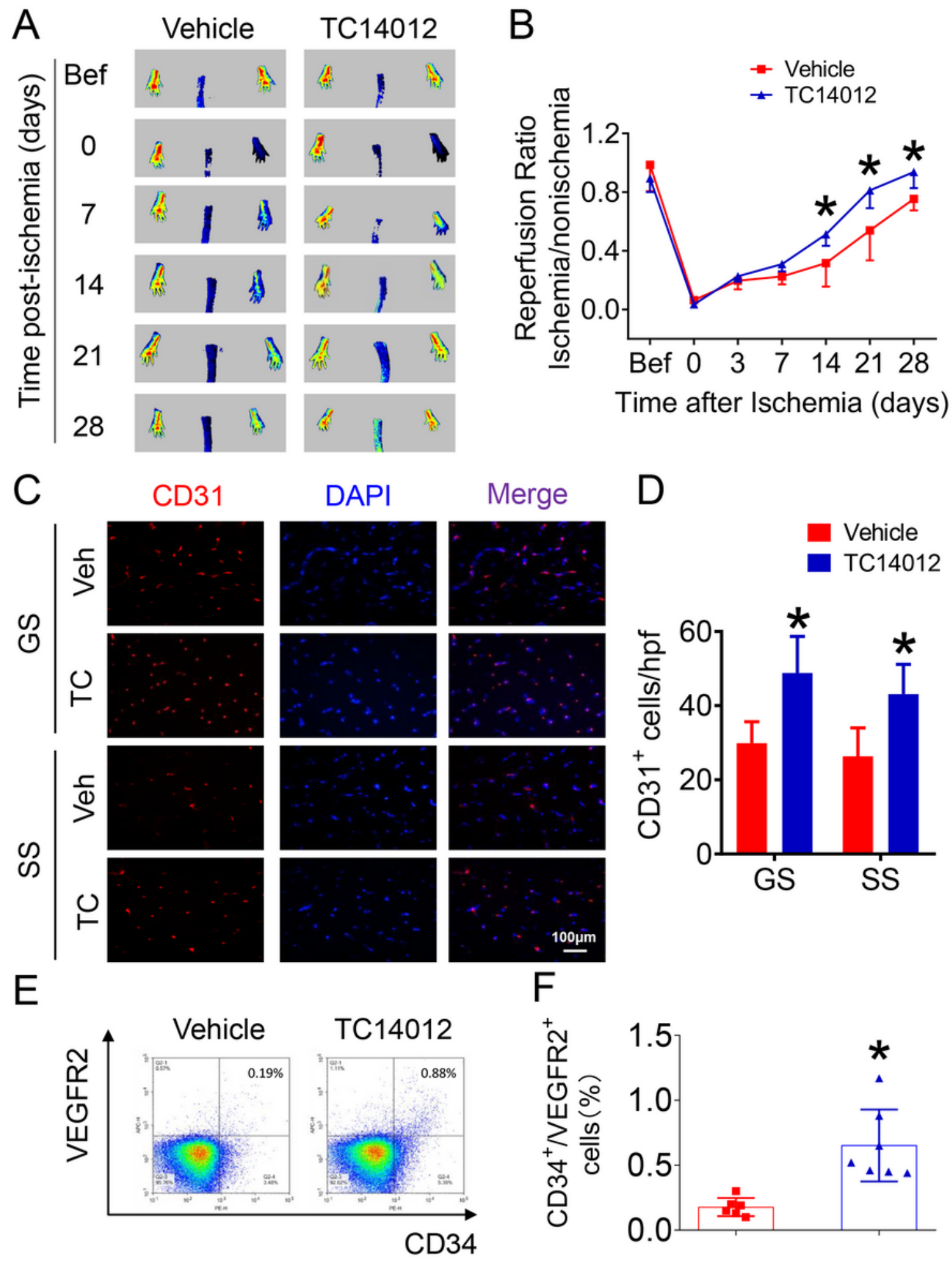

Figure 8

TC14012 improves blood perfusion recovery and angiogenesis in ischemic limb of $d b / d b$ diabetic mice.

Real-time blood perfusion is detected and visualized by a Pericam Perfusion Speckle Imager (PSI), the images of blood perfusion were shown at before (Bef), 0, 7, 14, 21, 28 days after HLI (A) and quantified by Image $J$ and expressed as the reperfusion ratio of ischemic hind limb to the contralateral non-ischemic hind limb (B). Antibody against CD31 was used to stained capillaries and 4',6-diamidino-2phenylindole 
(DAPI) was used to recognized nuclei in the transverse sections of soleus muscle (SS) and gastrocnemius muscle (GS) tissue from ischemic hind limbs (C). Capillary density was shown as the number of CD31-positive capillaries per high-power field (HPF, D). At $1 \mathrm{~h}$ after HLI surgery, mice with or without TC14012 (10 mg/kg body weight) treatment for $1 \mathrm{~h}$ were euthanized and the peripheral blood was collected to evaluate the percentage of EPCs $\left(\right.$ CD34 ${ }^{+} /$VEGFR2 $\left.{ }^{+}\right)$in circulation by a flow cytometry assay $(\mathrm{E}, \mathrm{F})$. Data were expressed as Mean $\pm \mathrm{SD}$. $\mathrm{n} \geq 6$ mice per group. ${ }^{*} P<0.05$ vs. Vehicle.

\section{Supplementary Files}

This is a list of supplementary files associated with this preprint. Click to download.

- FigS1.tif 